Louisiana State University

LSU Digital Commons

$12-1-2020$

\title{
Reconstructing genomes of carbon monoxide oxidisers in volcanic deposits including members of the class ktedonobacteria
}

\author{
Marcela Hernández \\ Thünen Institute of Biodiversity \\ Blanca Vera-Gargallo \\ University of Seville \\ Marcela Calabi-Floody \\ Universidad de la Frontera \\ Gary M. King \\ Louisiana State University \\ Ralf Conrad \\ Max Planck Institute for Terrestrial Microbiology
}

See next page for additional authors

Follow this and additional works at: https://digitalcommons.Isu.edu/biosci_pubs

\section{Recommended Citation}

Hernández, M., Vera-Gargallo, B., Calabi-Floody, M., King, G., Conrad, R., \& Tebbe, C. (2020). Reconstructing genomes of carbon monoxide oxidisers in volcanic deposits including members of the class ktedonobacteria. Microorganisms, 8 (12), 1-17. https://doi.org/10.3390/microorganisms8121880 


\section{Authors}

Marcela Hernández, Blanca Vera-Gargallo, Marcela Calabi-Floody, Gary M. King, Ralf Conrad, and Christoph C. Tebbe 


\title{
Reconstructing Genomes of Carbon Monoxide Oxidisers in Volcanic Deposits Including Members of the Class Ktedonobacteria
}

\author{
Marcela Hernández ${ }^{1,2,3, * \mathbb{C}}$, Blanca Vera-Gargallo ${ }^{4}$, Marcela Calabi-Floody ${ }^{5}$, Gary M. King ${ }^{6}$, \\ Ralf Conrad $^{3}$ and Christoph C. Tebbe ${ }^{1}$ (D) \\ 1 Johann Heinrich von Thünen Institut, Institut für Biodiversität, 50, 38116 Braunschweig, Germany; \\ christoph.tebbe@thuenen.de \\ 2 School of Environmental Sciences, Norwich Research Park, University of East Anglia, Norwich NR4 7TJ, UK \\ 3 Max Planck Institute for Terrestrial Microbiology, 35043 Marburg, Germany; conrad@mpi-marburg.mpg.de \\ 4 Department of Microbiology and Parasitology, Faculty of Pharmacy, University of Seville, \\ 41012 Seville, Spain; vera@us.es \\ $5 \quad$ BIOREN-UFRO, Universidad de La Frontera, Temuco 4811230, Chile; marcela.calabi@ufrontera.cl \\ 6 Department of Biological Sciences, Louisiana State University, Baton Rouge, LA 70803, USA; \\ gkingme@gmail.com \\ * Correspondence: marcela.hernandez@uea.ac.uk
}

Received: 28 October 2020; Accepted: 24 November 2020; Published: 27 November 2020

\begin{abstract}
Microorganisms can potentially colonise volcanic rocks using the chemical energy in reduced gases such as methane, hydrogen $\left(\mathrm{H}_{2}\right)$ and carbon monoxide (CO). In this study, we analysed soil metagenomes from Chilean volcanic soils, representing three different successional stages with ages of 380, 269 and 63 years, respectively. A total of 19 metagenome-assembled genomes (MAGs) were retrieved from all stages with a higher number observed in the youngest soil (1640: 2 MAGs, 1751: 1 MAG, 1957: 16 MAGs). Genomic similarity indices showed that several MAGs had amino-acid identity (AAI) values $>50 \%$ to the phyla Actinobacteria, Acidobacteria, Gemmatimonadetes, Proteobacteria and Chloroflexi. Three MAGs from the youngest site (1957) belonged to the class Ktedonobacteria (Chloroflexi). Complete cellular functions of all the MAGs were characterised, including carbon fixation, terpenoid backbone biosynthesis, formate oxidation and CO oxidation. All 19 environmental genomes contained at least one gene encoding a putative carbon monoxide dehydrogenase (CODH). Three MAGs had form I coxL operon (encoding the large subunit CO-dehydrogenase). One of these MAGs (MAG-1957-2.1, Ktedonobacterales) was highly abundant in the youngest soil. MAG-1957-2.1 also contained genes encoding a [NiFe]-hydrogenase and hyp genes encoding accessory enzymes and proteins. Little is known about the Ktedonobacterales through cultivated isolates, but some species can utilise $\mathrm{H}_{2}$ and $\mathrm{CO}$ for growth. Our results strongly suggest that the remote volcanic sites in Chile represent a natural habitat for Ktedonobacteria and they may use reduced gases for growth.
\end{abstract}

Keywords: Ktedonobacteria; carbon monoxide dehydrogenase; metagenome-assembled-genome; volcanic soils; shotgun sequencing

\section{Introduction}

Volcanic eruptions provide a model for understanding soil-forming processes and the roles of pioneer bacteria during early biotic colonisation. Recently, it has been demonstrated that the structure of microbial communities can play a key role in the direction of plant community succession pathways [1]. This is due in part to bacterial contributions to weathering of volcanic rocks, which releases nutrients, resulting in some of the most fertile soils in the world. 
After lava and other volcanic deposits (i.e., ash and tephra) cool sufficiently, mineral surface areas become accessible for microbial colonisation [2-5]. In fact, microbes, and especially bacteria, are among the first colonisers of volcanic deposits and thereby initiate soil formation during the early stages of terrestrial ecosystem development [6-12]. While methane $\left(\mathrm{CH}_{4}\right)$, hydrogen sulphide $\left(\mathrm{H}_{2} \mathrm{~S}\right)$, hydrogen $\left(\mathrm{H}_{2}\right)$ and carbon monoxide $(\mathrm{CO})$ have been proposed to promote bacterial colonisation and support microbial life in these organic-carbon-deficient environments $[13,14]$, the actual carbon and energy sources of the first colonisers remain elusive, but likely include a range of endogenous and exogenous sources, including reduced minerals and gases. The microbial ability to utilise these substrates for growth, and thereby initiate the formation of soil organic material, depends on specialised enzymes which may not be prevalent in many different microbial groups, thus representing a limited phylogenetic distribution. As a consequence, this could constrain the composition of pioneering microbial communities.

$\mathrm{CO}$ is a potential source of carbon and energy for microbes pioneering the colonisation of volcanic substrates. $\mathrm{CO}$ utilisation under oxic conditions requires a molybdenum-dependent carbon monoxide dehydrogenase (Mo-CODH), which catalyses the oxidation of $\mathrm{CO}$ to $\mathrm{CO}_{2}$ [15]. Surveys of genome databases (e.g., Integrated Microbial Genomes) reveal that Mo-CODHs occur in Proteobacteria, Actinobacteria, Firmicutes, Chloroflexi, Bacteroidetes, Deinococcus-Thermus, Halobacteria and Sulfolobales, among others. They were originally described as inducible enzymes and have subsequently been shown to be upregulated by carbon limitation during growth of Actinobacteria (e.g., Rhodococcus and Mycobacterium smegmatis [15-17]), and Chloroflexi (e.g., Thermogemmatispora and Thermomicrobium [18]). Mo-CODH has been previously targeted in molecular ecological studies of $\mathrm{CO}$ oxidisers in volcanic systems and other extreme environments [15]. These studies have revealed changes in community composition with the age and developmental status of individual sites [7,15]. The results suggest that $\mathrm{CO}$-oxidising communities are not static, but that they change in response to changing environmental conditions, and possibly affect the direction of changes.

In a preceding study, we identified the microbial communities involved in volcanic soil formation in different sites on Llaima Volcano (Chile). The bacterial communities of soils from 3 sites affected by lava deposition in 1640, 1751 and 1957 were analysed using 16S rRNA gene amplicon sequencing [19] and it was demonstrated that microbial diversity increased with the age of the soil deposits. Interestingly, bacterial phylotypes of the poorly studied Ktedonobacterales were among the predominant community members in the 1957 soil, representing 37\% of all OTUs, as compared with $18 \%$ in the 1751 and $7 \%$ in the 1640 soils. Thus, we suspected that bacteria of this order could be instrumental for the initiation of soil formation, paving the way for soil organic carbon formation and preparing a substrate for microbial colonisation and plant growth. Some already cultivated Ktedonobacterales were found to be carboxydotrophs and hydrogenotrophs (i.e., carbon monoxide (CO) and hydrogen $\left(\mathrm{H}_{2}\right)$ oxidisers/consumers) [14]. Thus, this leads to the hypothesis that $\mathrm{CO}$ and $\mathrm{H}_{2}$ are important carbon and energy sources for early stages of microbes colonising the Llaima Volcano soil. However, the ecophysiology of the few bacterial isolates assigned to Ktedonobacterales limits predictions about metabolic functions based on 16S rRNA gene sequences alone.

Therefore, in this study, a metagenomic approach was chosen to identify microbial traits associated with early stages of colonisation and soil formation in a volcanic ecosystem. In particular, the presence of functional genes implicated in CO-oxidation (coxL genes) and $\mathrm{H}_{2}$-oxidation (hyd and hyp genes) was assessed from metagenome-assembled genomes (MAGs). These MAGs were retrieved from volcanic soils of different ages, representing sites 1640,1751 and 1957. During this period, the soil formation evolved as indicated by their different levels of soil organic matter ranging from $65.33 \%$ in the most recent soil (1957) to 9.33\% in both medium (1751) and oldest soils (1640) [20].

Especially, the most recent soil (youngest soil) was suspected to reveal microbial adaptations to the challenging environmental conditions and thus to unveil the metabolic processes which initiate microbial colonisations. Therefore, functional metabolic modules annotated in the environmental genomes were analysed, with a main focus on the poorly characterised class of the Ktedonobacteria 
(Chloroflexi). Three Ktedonobacteria MAGs were obtained and all contained genes encoding CO and $\mathrm{H}_{2}$ oxidation. Additional MAGs from other phyla were also found to contain these genes. Our study advances the understanding of the ecology of Ktedonobacteria and their potential to act as early colonisers in volcanic soils.

\section{Materials and Methods}

\subsection{Sequencing}

The DNA from the volcanic soils used in our study had been previously extracted [20]. The soil physico-chemical characteristics have been published [20] showing a pH of 5.6 in both the medium and oldest soil and 4.7 in the youngest soil, and nitrogen (mg/kg) of 25 (1640), 26 (1751) and 36 (1957). Briefly, the soil samples originated from three different sites of different ages according to the latest lava eruption (1640,1751, 1957, map in Reference [20]). A total of nine samples (triplicate per site) were sequenced on an Illumina MiSeq at the Max-Planck-Genome Centre, Köln, Germany. The metagenome was analysed on a high-performance computer using 650 GB RAM and 64 cores at the Thünen Institute of Biodiversity, Braunschweig, Germany.

\subsection{Quality Control}

The sequence reads were checked using FastQC version 0.11.8 [21]. Low-quality reads were discarded using BBDuk version 38.68, quality-trimming to Q15 using the Phred algorithm [22]. A schematic overview of the steps and programs used are shown in Figure 1.

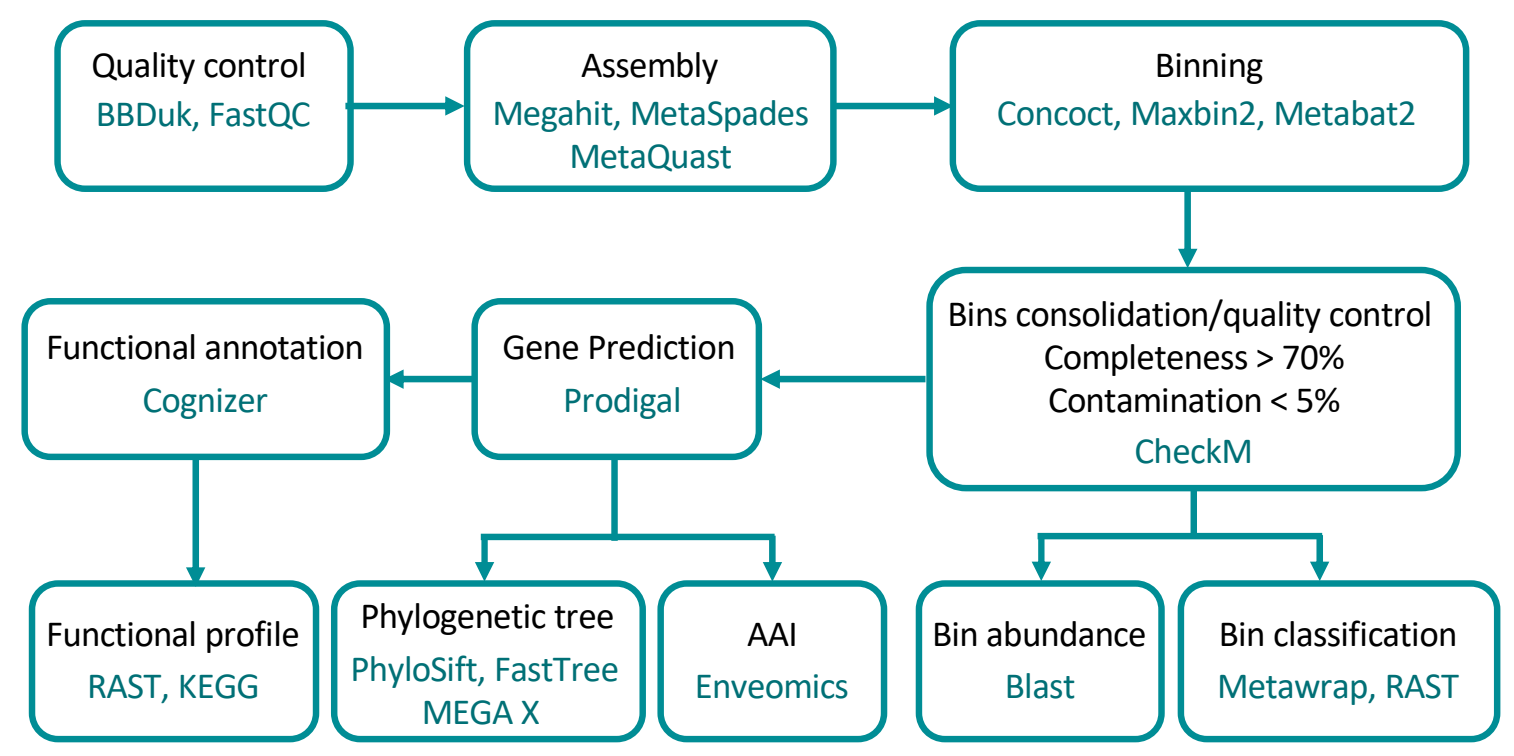

Figure 1. Workflow of the metagenome analysis and programs used in the present study. Binning was run by using the MetaWrap package. AAI: Amino Acid Identity.

\subsection{Metagenome Assembly and Binning}

All trimmed Illumina reads were merged into longer contiguous sequences (scaffolds) using de novo assemblers Megahit version 1.2.8 [23] with k-mers 21, 29, 39, 59, 79, 99, 119 and 141, and MetaSPAdes (SPAdes for co-assembly) version 3.13.1 [24,25] with k-mers 21, 31, 41, 51, 61, 71 and 81. Triplicate samples were co-assembled in order to improve the assembly of low-abundance organisms. Assembly quality was checked with MetaQuast version 5.0.2 [26], showing that the best quality was obtained with SPAdes for our samples (data not shown). Downstream analysis was carried out using the scaffolds retrieved from SPAdes. Krona charts [27] were recovered from MetaQuast runs 
to identify taxonomic profiles. Downstream binning analysis was performed with two sets of scaffolds: full-size scaffolds and scaffolds larger than $1000 \mathrm{bp}$.

Metagenomic binning of the assembled scaffolds was carried out with the metaWRAP version 1.2.1 pipeline [28], in which binning module employs three binning software programs: MaxBin2 [29], metaBAT2 [30], and CONCOCT [31]. Completion and contamination metrics of the extracted bins were estimated using CheckM [32]. The resulting bins were collectively processed to produce consolidated metagenome-assembled genomes (MAGs) using the bin_refinement module (criterion: completeness $>70 \%$; contamination < 5\%). Both sets of MAGs (18 from scaffolds larger than $1000 \mathrm{bp}$ and 17 from full-size scaffolds) were aggregated, visualised with VizBin [33] and then dereplicated using dRep [34]. Only the highest scoring MAG from each secondary cluster was retained in the dereplicated set. The abundance of each MAG in the different sites was calculated using BLASTN version 2.5.0+ [35], keeping only hits with $>95 \%$ identity and e-value 1 e- 5 for the analysis [36]. A final heatmap was constructed using the function heatmap.2 from the gplots package version 3.0.4 [37] in $R$ version 4.0.2 (https://www.r-project.org).

\subsection{Functional Annotation}

The open reading frames (ORFs) in all scaffolds of each MAG were predicted using Prodigal (v2.6.3) [38]. Functions were annotated using Cognizer [39] and the Kyoto Encyclopedia of Genes and Genomes (KEGG) annotation framework [40]. The annotations of the predicted proteins from KEGG were used to confirm protein functional assignment and identify pathways. Complete pathways were identified using KEGG BRITE pathway mapping [40]. Aerobic carbon-monoxide dehydrogenases and hydrogen dehydrogenase were also identified using KEGG ortholog annotations. CODH was further distinguished as form I and form II (putative CODH) based on active site motifs present in coxL genes (e.g., Reference [41]).

\subsection{Phylogenomic Analysis}

Taxonomic classification of MAGs was performed using the classify_bins module from metaWRAP which relies on the NCBI_nt database. MAGs were also screened using the RAST Server (Rapid Annotations using Subsystems Technology [42,43]), which also allowed to retrieve information regarding close relative genomes in order to construct the phylogenetic tree.

To estimate intergenomic similarity, amino-acid comparisons between MAGs and their closest relative genomes present in the databases were calculated based on reciprocal best hits (two-way AAI) using the enveomics collection (http://enve-omics.gatech.edu/ [44]).

The phylogenetic affiliation of MAGs was determined by constructing a genomic tree using FastTree version 2.1.11 [45]. Reference genomes were manually downloaded from the National Center for Biotechnology Information (NCBI) Refseq database (Supplementary Table S1). Conserved genes from the extracted bins and the reference genomes were concatenated using Phylosift version 1.0.1 [46].

Phylogenetic analysis of the large sub-unit CO dehydrogenase gene $(\operatorname{coxL})$ using the Maximum Likelihood method with a JTT matrix-based model [47] was performed. Bootstrap values (100 replicates) are shown where support $\geq 70$ percent. The scale bar indicates substitutions per site. All gapped positions were deleted, resulting in 420 positions in the final dataset. Evolutionary analyses were conducted in MEGA X [48,49].

\subsection{Accession Number}

Raw metagenomic data and environmental genomes derived from binning processes were deposited in the Sequence Read Archive (SRA) under the bioproject accession number PRJNA602600 for raw data and PRJNA602601 for metagenome-assembled genomes. 


\section{Results}

\subsection{MAGs Recovery}

A total of $\sim 3-4$ million scaffolds were recovered from the soil metagenomes in each site. Even though all the sites underwent similar sequencing efforts (between 2.3 GB in 1640 and 1957 to $2.4 \mathrm{~GB}$ in 1751), the youngest soil had the largest number of scaffolds with 499 sequences $>50 \mathrm{~kb}$ (N50 length of 700) compared to the oldest soil with only 12 scaffolds with a size $>50 \mathrm{~kb}$ (N50 length of 914) (Table 1). A total of 19 MAGs with a completeness of $>70 \%$ and a contamination $<5 \%$ ( 2 from 1640, 1 from 1751 and 16 from 1957) were retrieved and characterised.

Table 1. Summary report for the assembly quality assessment using MetaQuast.

\begin{tabular}{|c|c|c|c|}
\hline Statistics & 1640 & 1751 & 1957 \\
\hline Number of scaffolds (>=0 bp) & $3,631,380$ & $4,047,900$ & $3,138,527$ \\
\hline Number of scaffolds ( $>=500 \mathrm{bp}$ ) & $1,488,437$ & $1,609,020$ & $1,578,887$ \\
\hline Number of scaffolds ( $>=1000 \mathrm{bp})$ & 326,980 & 333,885 & 447,418 \\
\hline Number of scaffolds ( $>=25,000 \mathrm{bp}$ ) & 172 & 195 & 1551 \\
\hline Number of scaffolds ( $>=50,000 \mathrm{bp}$ ) & 12 & 40 & 499 \\
\hline Total length $(>=0 \mathrm{bp})$ & $2,320,437,372$ & $2,460,323,814$ & $2,396,069,091$ \\
\hline Total length $(>=500 \mathrm{bp})$ & $1,385,386,471$ & $1,404,094,641$ & $1,717,252,425$ \\
\hline Total length $(>=1000 \mathrm{bp})$ & $631,179,105$ & $570,404,360$ & $964,085,094$ \\
\hline Total length $(>=25,000 \mathrm{bp})$ & $5,697,241$ & $8,152,100$ & $8,236,5310$ \\
\hline Total length $(>=50,000 \mathrm{bp})$ & 686,375 & $2,983,958$ & $46,419,897$ \\
\hline N50 & 914 & 850 & 700 \\
\hline L50 & 391,387 & 477,193 & 348,604 \\
\hline
\end{tabular}

N50-length such that scaffolds of this length or longer include half the bases of the assembly; L50-number of scaffolds that are longer than, or equal to, the N50 length and therefore include half the bases of the assembly (https://www.ncbi.nlm.nih.gov/assembly/help/\#globalstats).

\subsection{MAG Identification}

MAGs were affiliated to the phyla Actinobacteria, Proteobacteria, Acidobacteria, Gemmatimonadetes, Chloroflexi, Firmicutes and Verrucomicrobia (Figure 2). In the oldest soil, two environmental genomes were retrieved related to Actinomycetales (Actinobacteria) and Rhodospirillales (Proteobacteria). The only MAG retrieved from the middle soil was related to Acidobacteria. MAGs binned from the youngest soil included six assigned to Acidobacteria, one to Proteobacteria, one to Firmicutes, three to Actinobacteria, one to the phylum Gemmatimonadetes, one to Verrucomicrobia and three to the phylum Chloroflexi (Figure 2).

The abundance of the MAGs in each site was calculated by using BLASTN (Figure 3). MAGs were more abundant from the soil they were recovered. MAGs with a total abundance $>1 \%$ were found only in the young soil (1957). MAG 1957-2.1 (Ktedonobacteria, 1.21\% \pm 0.82\%), MAG 1957-5.1 (Actinomycetales, 1.02\% \pm 0.84\%), MAG 1957-13.1 (Verrucomicrobiales, 1.58\% $\pm 1.1 \%$ ) and MAG 1957-16.1 (Acidobacteria, 1.14\% \pm 0.87\%) were the most abundant MAGs (Figure 3). 


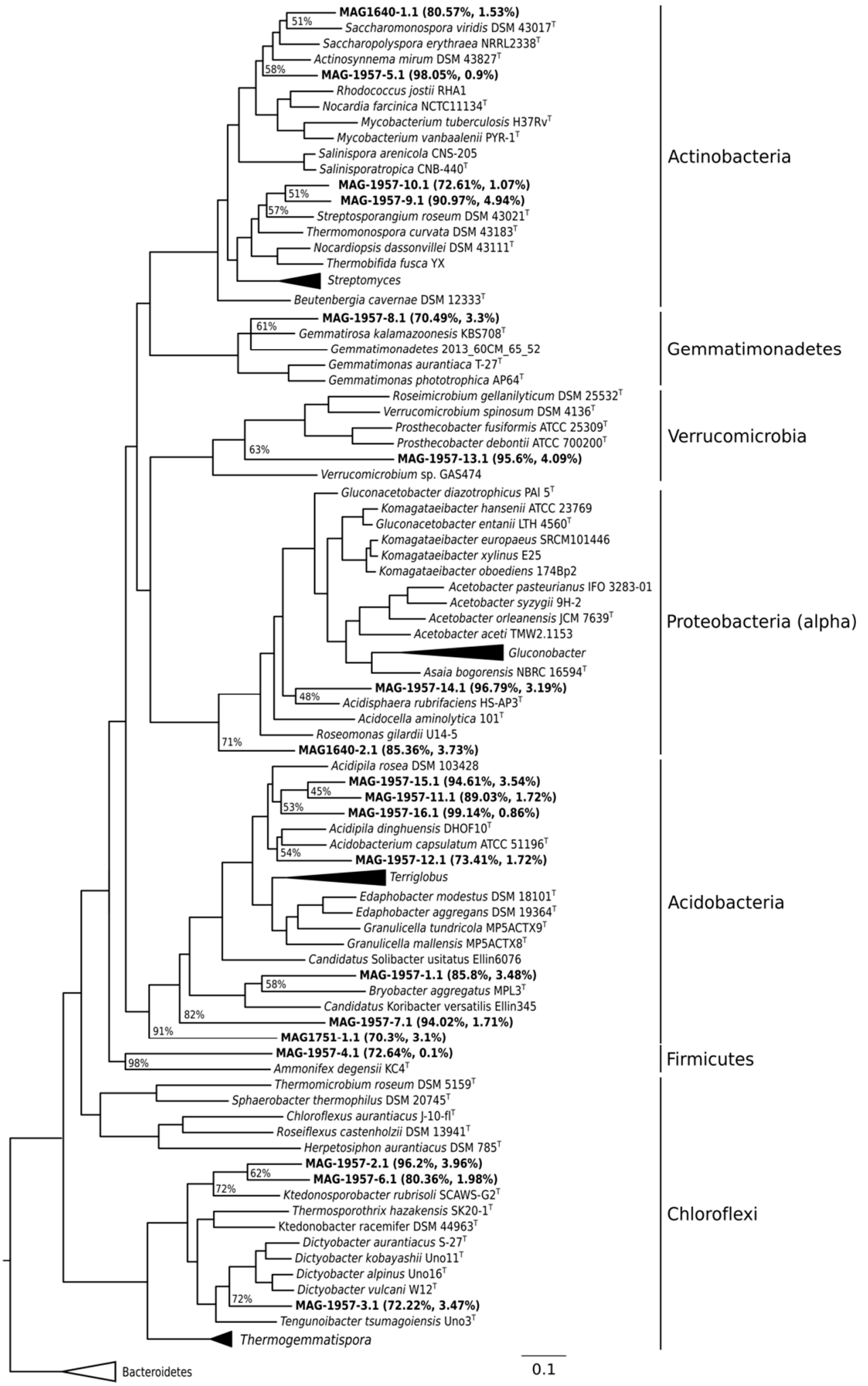

Figure 2. Phylogenomic tree of the bacterial genomic bins. The tree was built with PhyloSift against reference genomes downloaded from NCBI. MAGs are indicated in bold together with their respective completeness and contamination. FastTree confidence values of MAG branches are shown. The horizontal bar represents $10 \%$ sequence divergence. 


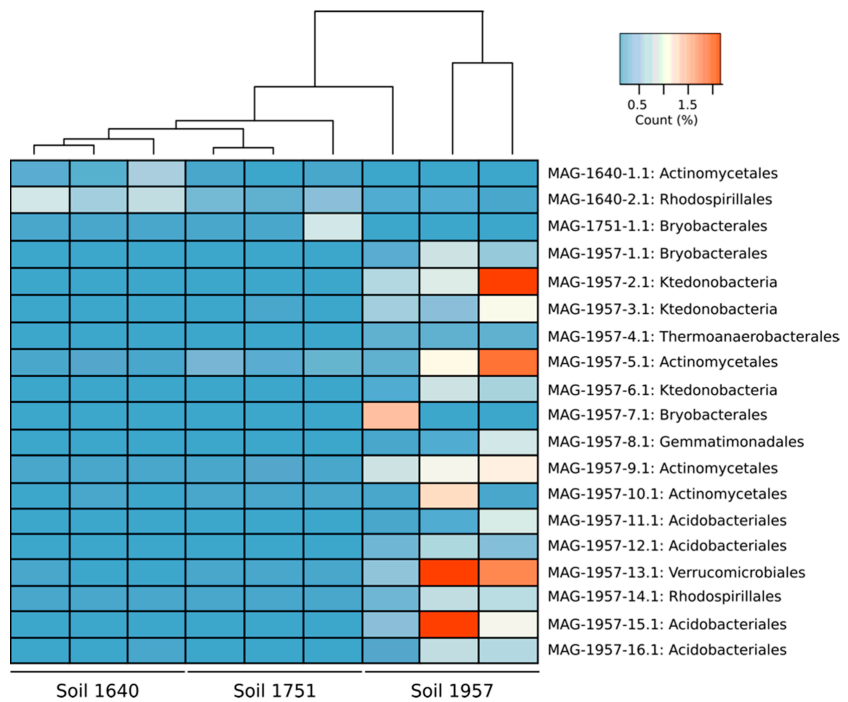

Figure 3. Heatmap representing the abundance of MAGs in each metagenome. The analysis was done by blast and only hits greater than $95 \%$ identity and e-value $1 \times 10^{-5}$ were used.

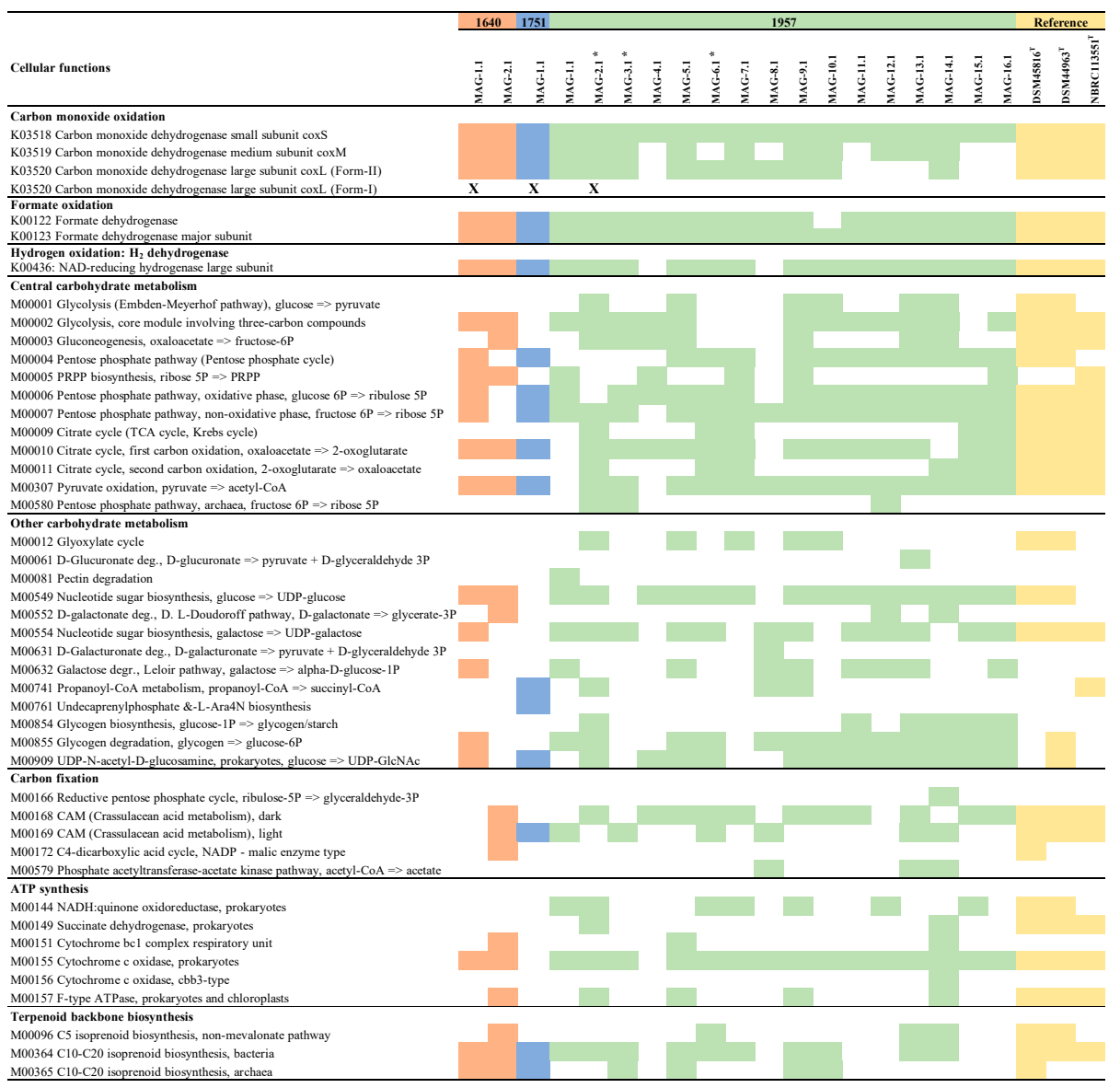

Figure 4. Summary of complete cellular functions and other high-level features in the MAGs recovered form sites 1640, 1751 and 1957 retrieved from KEGG analysis. Reference genomes for Ketedonobacteria: DSM45816T [50], DSM44963T [51] and NBRC 113551T [52], (K: KEGG orthology; M: KEGG Mode). Asterisks indicate the MAGs isolated from the class Ktedonobacteria. " $\mathrm{X}$ " indicates MAGs containing genes encoding form I of the CoxL. 


\subsection{Metabolic Characterisation of MAGs}

Genes encoding enzymes involved in carbohydrate and energy metabolism, such as carbon fixation, sulphur metabolism, ATP synthesis and nitrogen metabolism, as well as terpenoid backbone biosynthesis, were found in all the MAGs (Figure 4). Other functions, including xenobiotic biodegradation, fatty acid metabolism, nucleotide metabolism and vitamin metabolism, among others, were also found (Supplementary Table S2).

\subsubsection{Characterisation of CODH and Hydrogenase Genes in MAGs}

Three MAGs (MAG-1640-1.1, MAG-1751-1.1 and MAG-1957-2.1) encoded form I of the CO-dehydrogenase large subunit (coxL). These MAGs were each associated with a particular soil, with low abundance in the metagenomes of the other sites (Figure 5A). In addition to these three form-I coxL-encoding MAGs, 15 other scaffolds from MAGs containing form II coxL-like genes were recovered (data not shown), but the function of form II CoxL is not yet known. The arrangement of genes encoding form I CODH in each of the MAGs is shown in Figure 5B. It should be noted that all of these three MAGs show the canonical arrangement for the three structural genes of $\mathrm{CODH}$, that is the MSL ( $\operatorname{coxM}-\operatorname{coxS}-\operatorname{coxL}$ ) genes. The genes encoding the [NiFe]-hydrogenase and its accessory proteins were only identified in MAG-1957-2.1, and instead, only some of the accessory hyp genes were found in the other two MAGs (Figure 5B). A phylogenetic analysis of the form I coxL genes was performed, showing they are affiliated with Actinobacteria (MAG-1640-1.1), Nitrospirae Candidatus Manganitrophus noduliformans (MAG-1751-1.1) and Chloroflexi (MAG-1957-2.1) (Figure 6). This grouping is consistent with the results of PhyloSift (Figure 2), except for MAG-1751-1.1, where it was loosely associated with Acidobacteria (although with an amino-acid identity of only $40 \%$ ) rather than Nitrospirae.

\subsubsection{Complete Metabolic Characterisation of Ktedonobacterales MAGs}

Here, we focused on the Ktedonobacterales MAGs because of their apparent importance in early soil formation. Three Ktedonobacterales MAGs were identified in the 1957 soil metagenomes but were not found in the older soils (Figures 2 and 3). Two of the MAGs (MAGs 1957-2.1 and 1957-3.1), affiliated to the class Ktedonobacteria (phylum Chloroflexi), contained genes for the complete electron transport chain, citric acid metabolism, nitrogen metabolism, sulphur metabolism, several transporters, the complete gene set for carbon monoxide oxidation (CO dehydrogenase), herbicide degradation and degradation aromatics, as well as the major subunit of the formate dehydrogenase, and also a hydrogenase. MAG 1957-6.1 (Ktedonobacteria) had very similar pathways as the other Chloroflexi MAGs, except a step for CO-oxidation and the electron transport chain were absent (Figure 4, Supplementary Table S2, Figure 7). 
A.

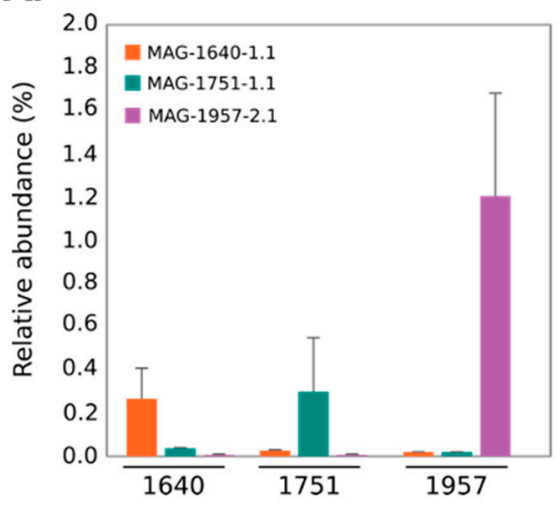

B.

\section{MAG-1640-1.1}
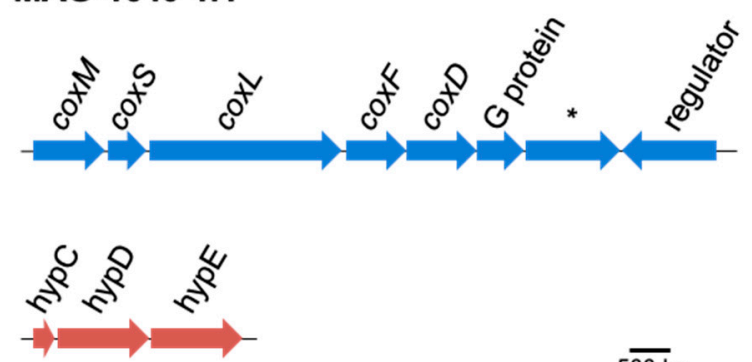

$5 \overline{00 \mathrm{bp}}$

MAG-1751-1.1
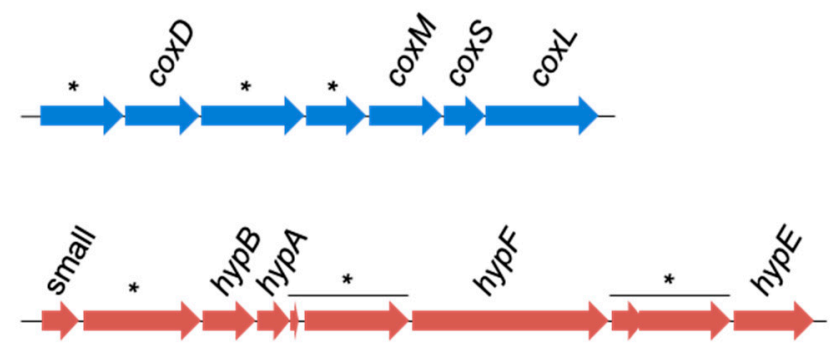

$5 \overline{00 \mathrm{bp}}$

MAG-1957-2.1
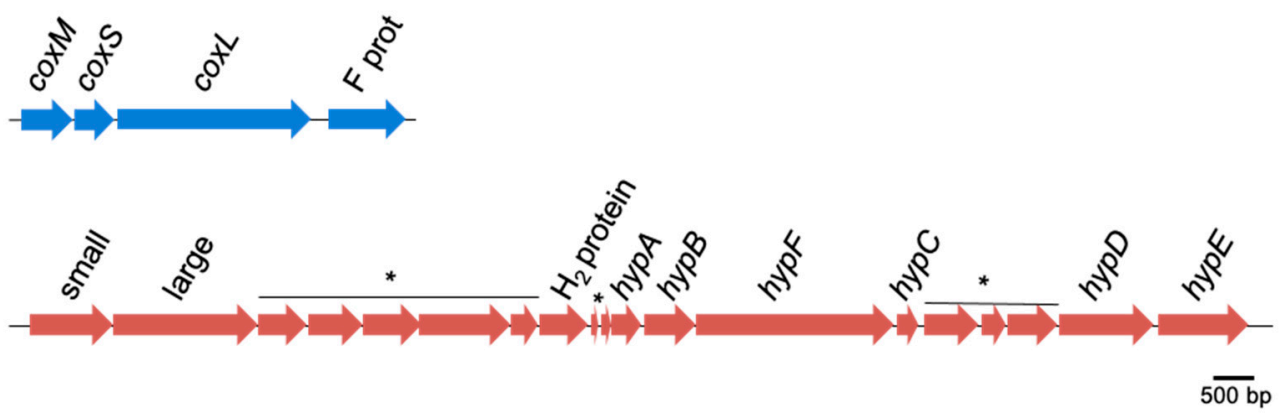

Figure 5. (A) Relative abundance of MAG-1640-1.1, MAG-1751-1.1 and MAG-1957-2.1 in sites 1640, 1751 and 1957. Bars indicate standard error of triplicates. (B) Gene arrangement of the carbon monoxide dehydrogenase (CODH) and membrane-bound [NiFe]-hydrogenase in MAG-1640-1.1, MAG-1751-1.1 and MAG-1957-2.1 (* indicates hypothetical proteins; small indicates NiFe-hydrogenase small subunit and large indicates NiFe-hydrogenase large subunit). 


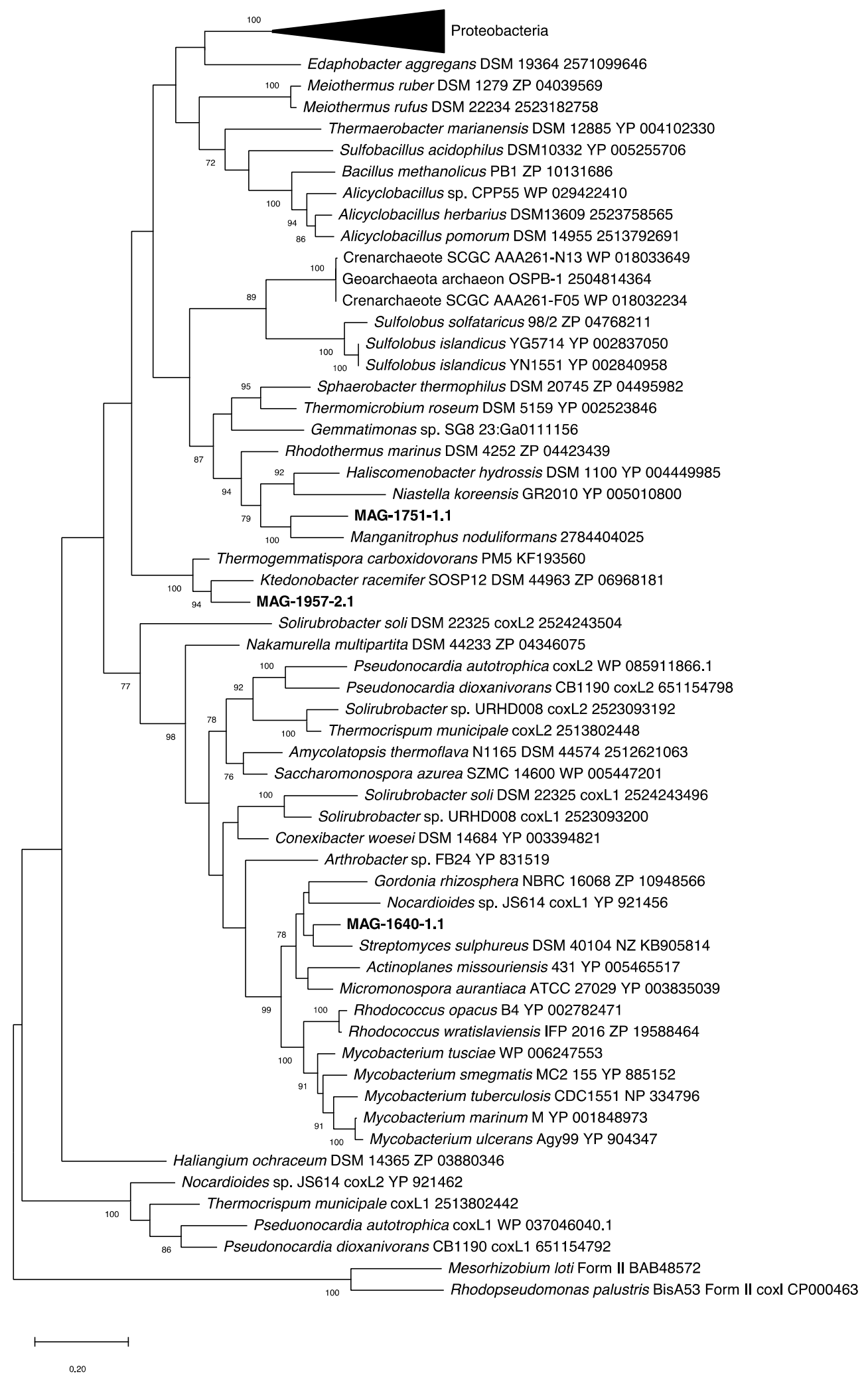

Figure 6. Phylogenetic tree of form I carbon monoxide dehydrogenase large subunit (CoxL) of metagenome-assembled genomes retrieved from Llaima volcano (MAG-1640-2.1, MAG-1751-1.1 and MAG-1957-2.1) against reference sequences (with accession numbers included in the tree). The tree was drawn using the Maximum Likelihood method using MEGA X [48]. Bootstrap values (500 replications) are shown at the nodes. MAG-1751_1.1 is a partial sequence of the coxL gene. 


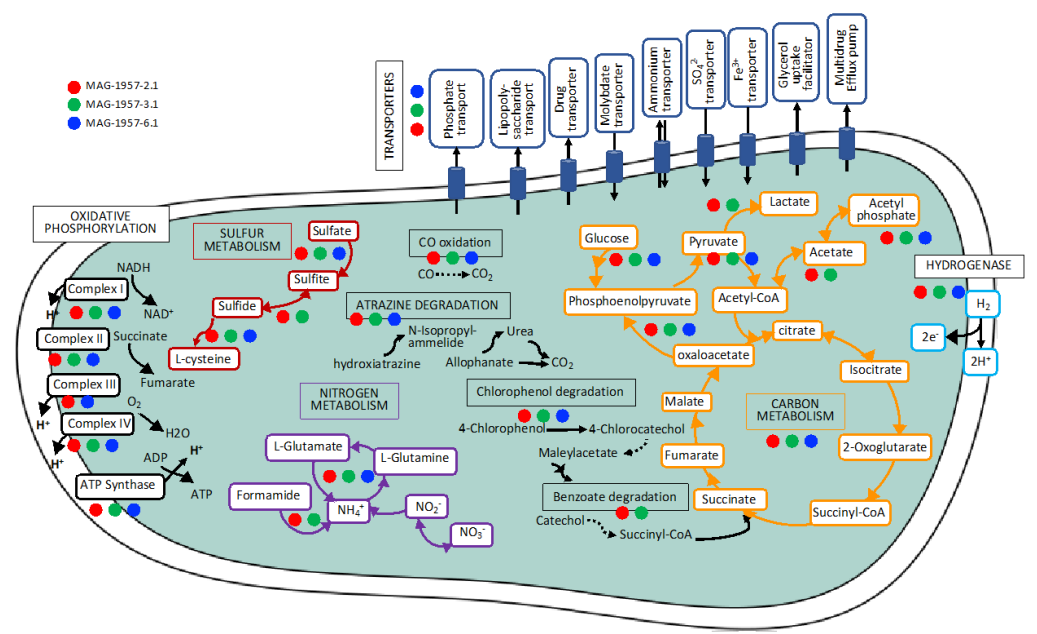

Figure 7. Metabolic reconstruction for some of the most important functions in the Ktedonobacteria MAGs isolated from the youngest soil using KEGG (more information in Figure 4 and Supplementary Table S2), dashed lines were used for incomplete pathways. Multi-arrow lines indicate several steps of a pathway.

\section{Discussion}

\subsection{Characterisation of MAGs}

In this study, a characterisation of metagenome-assembled genomes retrieved from Llaima volcano was performed. This study builds from a previous study [19] in which 16S rRNA gene amplicon-based sequences from those soils were analysed. The main objective of this study was to characterise genomes from those sites and to analyse the functions of the abundant but the poorly characterised Ktedonobacteria (phylum Chloroflexi) present at Llaima volcano. The relative abundance of the main phyla based on classification of scaffolds larger than $500 \mathrm{bp}$ showed that microbial communities change as the soils age (Supplementary Figure S1). This corroborates findings from a previous study [19]. For example, the relative abundance of Chloroflexi is higher in the younger soils ( $28 \%$ in the youngest soil to $7 \%$ in the oldest soil) and the opposite trend is observed for members of the phylum Proteobacteria, as their abundance increases as the soil ages (from $42 \%$ in the youngest soil to $59 \%$ in the oldest soil) (Supplementary Figure S1). Except for those related to Firmicutes and Verrucomicrobia, and to a lesser extent Acidobacteria and Proteobacteria, the extracted environmental genomes had an amino acid identity $>50 \%$ with their closest reference genome (Supplementary Table S3), which suggest that they belonged to those genera [53].

A total of 16 MAGs were recovered from the youngest soil (1957) (Figure 3). This soil is only partially vegetated (about $5 \%$ ) by mosses and lichens. The microbial community in this area likely harbours populations able to grow as facultative chemolithoautotrophs or mixotrophs on carbon monoxide, hydrogen or methane. This high relative abundance of MAGs with genes for $\mathrm{CO}$ and hydrogen utilisation in the youngest soils is consistent with reports by King and colleagues for Hawaiian and Japanese volcanic deposits (21- to 800-year-old sites). For some of those sites, microbial community structure changed as the soil matured, with members of the phylum Proteobacteria dominating vegetated sites while younger sites were enriched with Ktedonobacteria within the Chloroflexi and characterised by relatively high rates of atmospheric CO uptake $[7,14,54]$.

MAGs were most abundant in the soil site from where they were retrieved (Figure 3). Relatively few MAGs were retrieved from the two older soils, which can be explained by the higher diversity in these soils and the decreased likelihood of recovering MAGs from groups such as Actinobacteria, Acidobacteria and Chloroflexi that were less common in them. In fact, several of the MAGs retrieved had a low relative abundance within the soils (Figure 3), which is consistent with their relative abundance of 16S rRNA genes in these soils [19]. Binning at the strain level remains a technical 
challenge [55], with the chances of retrieving MAGs at a given sequencing effort being reduced with increasing microdiversity (intra-population genetic diversity) and overall community diversity [56]. We previously reported that as the soil recovered and vegetation established, the microbial population appeared to enlarge and become more diverse [19], which explains the lower number of MAGs retrieved from more mature soil (1640 sample), compared to the younger sites (1957).

\subsection{Metabolic Characterisation of MAGs}

The three MAGs containing form I coxL genes were found in an operon structure (Figure 5B) typical of known CO oxidisers [41]. Form I coxL has been definitively associated with CO oxidation at high concentrations and also at sub-atmospheric levels [41]. Thus, even at low abundance, the presence of these cox-containing MAGs strongly suggests a capacity for atmospheric $\mathrm{CO}$ uptake at all the sites.

Most of the complete functions found from the Ktedonobacteria MAGs were also found in three reference genomes: Ktedonobacter racemifer DSM 44963 [51], Thermogemmatispora carboxidivorans PM5, isolated from a geothermal biofilm on Kilauea Volcano, Hawaii (USA) [50], and Dictyobacter volcani W12 [52]. According to our genomic analyses, all of these reference strains possess formate-, $\mathrm{H}_{2}$-, and CO-dehydrogenases, as do the MAGs recovered in the present study. Burkholderia strains (phylum Proteobacteria) [57], members of the phylum Chloroflexi [14] and other members of the phyla Proteobacteria and Actinobacteria [58] have also been reported as CO-oxidisers in Hawaiian volcanic deposits. coxL genes encoding the large subunit of the $\mathrm{CO}$ dehydrogenase have been found in Proteobacteria species from Kilauea and Miyake-jima volcanoes [10,14,54].

The taxonomies of MAGs 1640-1.1 and 1957-2.1 were consistent for coxL (Figure 6) and phylogenomic analyses (Figure 2). In contrast, MAG-1751-1.1 clustered weakly with Acidobacteria based on genomic analysis (40\% amino acid identity with a reference genome, see Supplementary Table S3) but did not cluster with Candidatus Manganitrophus noduliformans as did the coxL sequence from this MAG.

Several strains from the class Ktedonobacteria have been isolated from different environments (Supplementary Table S4), but only Dictyobacter vulcani W12 [52] and Thermogemmatispora carboxidivorans PM5 [50] have been isolated from volcanic environments. So far, the class Ktedonobacteria contains only six genera and fifteen formally proposed species. Out of the 15 type strains, 11 genomes are available on RefSeq (Supplementary Table S2). The order Ktedonobacterales contains the type strains Ktedonobacter racemifer SOSP1-21 ${ }^{\mathrm{T}}$ [51,59], Dictyobacter aurantiacus S-27 ${ }^{\mathrm{T}}$ [60], Dictyobacter vulcani W12 ${ }^{\mathrm{T}}$ [52], Thermosporothrix hazakensis SK20-1 ${ }^{\mathrm{T}}$ [61], Thermosporothrix narukonensis $\mathrm{F}^{\mathrm{T}}{ }^{\text {[62], Ktedonosporobacter }}$ rubrisoli SCAWS-G2 ${ }^{\mathrm{T}}$ [63], Tengunoibacter tsumagoiensis Uno3 ${ }^{\mathrm{T}}$, Dictyobacter kobayashii Uno11 ${ }^{\mathrm{T}}$ and Dictyobacter alpinus Uno16 ${ }^{\mathrm{T}}$ [64]. The order Thermogemmatispora contains the species Thermogemmatispora aurantia A1-2 ${ }^{\mathrm{T}}$ [65], Thermogemmatispora argillosa A3-2 ${ }^{\mathrm{T}}$ [65], Thermogemmatispora onikobensis NBRC 111776 (unpublished, RefSeq Nr NZ_BDGT00000000.1), Thermogemmatispora onikobensis ONI-1 ${ }^{\mathrm{T}}$ [66], Thermogemmatispora foliorum $\mathrm{ONI}-5^{\mathrm{T}}$ [66] and Thermogemmatispora carboxidivorans $\mathrm{PM}^{\mathrm{T}}$ [50]. All of those genomes contain the complete gene set for carbon monoxide oxidation (CO dehydrogenase), as well as formate dehydrogenases and $\mathrm{H}_{2}$ dehydrogenases (Supplementary Table S4). Our study particularly brings more insights into the role that early colonisers of this group from volcanic soils may have in the development of soils.

The large subunit of the NAD-reducing hydrogenase was also found in several MAGs (Figure 4). Hydrogen metabolism has been shown to provide an additional energy source for some microorganisms and has been observed in bacteria and archaea [67]. Hydrogen dehydrogenases have also been found in members of the genus Cupriavidus (phylum Proteobacteria) from volcanic mudflow deposits in the Philippines, suggesting their potential contribution to hydrogen uptake [68].

\section{Conclusions}

This study is further evidence that poorly characterised groups, such as Ktedonobacteria, establish in remote volcanic sites and may use reduced gases for growth. Further studies are needed to demonstrate the activity of these pathways and their significance in volcanic deposits. 
Supplementary Materials: The following are available online at http://www.mdpi.com/2076-2607/8/12/1880/s1, Figure S1: Relative abundance of main phyla based on classification of scaffolds $>500 \mathrm{bp}$. Other phyla include members of Planctomycetes, Gemmatimonadetes, Cyanobacteria, Firmicutes, Armatimonadetes and Patescibacteria, Table S1: List of strains used for the tree construction, Table S2: Summary table of other complete cellular functions in the MAGs isolates form sites 1640, 1751 and 1957 retrieved from KEGG analysis. Table S3: Summary of the metagenome-assembled genomes (MAGs) isolated in the present study. Average amino-acid identity (AAI) was calculated by comparing the MAGs with their closest reference genomes identified based on the RAST results. The RefSeq accession number of the reference genomes are provided in parentheses, Table S4: Summary of enzymatic functions for $\mathrm{CO}-, \mathrm{H}_{2}-$, and formate-oxidation in the Ktedonobacteria reference genomes. Carbon monoxide oxidation: K03518 carbon monoxide dehydrogenase small subunit coxS, K03519 carbon monoxide dehydrogenase medium subunit coxM, K03520 carbon monoxide dehydrogenase large subunit coxL. Formate oxidation: K00122 formate dehydrogenase, K00123 formate dehydrogenase major subunit. Hydrogen oxidation: $\mathrm{H}_{2}$ dehydrogenase: K00436: NAD-reducing hydrogenase large subunit.

Author Contributions: Conceptualisation, M.H., R.C. and C.C.T.; sampling campaign, M.C.-F.; methodology, M.H. and B.V.-G.; software, M.H.; validation, M.H., B.V.-G., G.M.K., R.C. and C.C.T.; formal analysis, M.H.; investigation, M.H.; resources, M.H., R.C. and C.C.T.; data curation, M.H; writing-original draft preparation, M.H.; writing-review and editing, M.H., B.V.-G., G.M.K., R.C. and C.C.T.; visualisation, M.H.; supervision, R.C. and C.C.T.; project administration, M.H.; funding acquisition, M.H. All authors have read and agreed to the published version of the manuscript.

Funding: This research was funded by the Alexander von Humboldt Foundation, Germany.

Acknowledgments: We thank Stefan Mecke from the Thünen Institut für Biodiversität, Germany, for technical support. Part of the research presented in this paper was carried out on the High-Performance Computing Cluster supported by the Research and Specialist Computing Support Service (RSCSS) at the University of East Anglia. A special thanks to Mike Adams in RSCSS for technical support.

Conflicts of Interest: The authors declare no conflict of interest.

\section{References}

1. Wubs, E.R.; van der Putten, W.H.; Bosch, M.; Bezemer, T.M. Soil inoculation steers restoration of terrestrial ecosystems. Nat. Plants 2016, 2, 16107. [CrossRef] [PubMed]

2. Kelly, L.C.; Cockell, C.S.; Thorsteinsson, T.; Marteinsson, V.; Stevenson, J. Pioneer microbial communities of the Fimmvörðuháls Lava Flow, Eyjafjallajökull, Iceland. Microb. Ecol. 2014, 68, 504-518. [CrossRef] [PubMed]

3. Witt, V.; Ayris, P.M.; Damby, D.E.; Cimarelli, C.; Kueppers, U.; Dingwell, D.B.; Wörheide, G. Volcanic ash supports a diverse bacterial community in a marine mesocosm. Geobiology 2017, 15, 453-463. [CrossRef]

4. Smith, H.G. The colonization of volcanic tephra on deception island by protozoa. Br. Antarct. Surv. Bull. 1974, 38, 49-58.

5. Chadwick, O.A.; Derry, L.; Vitousek, P.M.; Huebert, B.J.; Hedin, L.O. Changing sources of nutrients during fourmillion years of ecosystem development. Nature 1999, 397, 491-497. [CrossRef]

6. Fujimura, R.; Sato, Y.; Nishizawa, T.; Nanba, K.; Oshima, K.; Hattori, M.; Kamijo, T.; Ohta, H. Analysis of early bacterial communities on volcanic deposits on the island of Miyake (Miyake-jima), Japan: A 6-year study at a fixed site. Microbes Environ. 2012, 27, 19-29. [CrossRef] [PubMed]

7. King, G.M. Contributions of atmospheric $\mathrm{CO}$ and hydrogen uptake to microbial dynamics on recent Hawaiian volcanic deposits. Appl. Environ. Microbiol. 2003, 69, 4067-4075. [CrossRef]

8. Ohta, H.; Ogiwara, K.; Murakami, E.; Takahashi, H.; Sekiguchi, M.; Koshida, K.; Someya, T.; Morishima, W.; Rondal, J.D.; Concepcion, R.N.; et al. Quinone profiling of bacterial populations developed in the surface layer of volcanic mudflow deposits from Mt. Pinatubo (the Philippines). Soil Biol. Biochem. 2003, 35, 1155-1158. [CrossRef]

9. Sato, Y.; Nishihara, H.; Yoshida, M.; Watanabe, M.; Rondal, J.D.; Ohta, H. Occurrence of hydrogen-oxidizing Ralstonia species as primary microorganisms in the Mt. Pinatubo volcanic mudflow deposits. Soil Sci. Plant Nutr. 2004, 50, 855-861. [CrossRef]

10. Gomez-Alvarez, V.; King, G.M.; Nüsslein, K. Comparative bacterial diversity in recent Hawaiian volcanic deposits of different ages. FEMS Microbiol. Ecol. 2007, 60, 60-73. [CrossRef]

11. Guo, Y.; Fujimura, R.; Sato, Y.; Suda, W.; Kim, S.W.; Oshima, K.; Hattori, M.; Kamijo, T.; Narisawa, K.; Ohta, H. Characterization of early microbial communities on volcanic deposits along a vegetation gradient on the island of Miyake, Japan. Microbes Environ. 2014, 29, 38-49. [CrossRef] [PubMed]

12. Kim, J.S.; Kim, D.S.; Lee, K.C.; Lee, J.S.; King, G.M.; Kang, S. Microbial community structure and functional potential of lava-formed Gotjawal soils in Jeju, Korea. PLoS ONE 2018, 13, e0204761. [CrossRef] [PubMed] 
13. King, G.M. Molecular and culture-based analyses of aerobic carbon monoxide oxidizer diversity. Appl. Environ. Microbiol. 2003, 69, 7257-7265. [CrossRef] [PubMed]

14. Weber, C.F.; King, G.M. Distribution and diversity of carbon monoxide-oxidizing bacteria and bulk bacterial communities across a succession gradient on a Hawaiian volcanic deposit. Environ. Microbiol. 2010, 12, 1855-1867. [CrossRef]

15. Cordero, P.R.F.; Bayly, K.; Leung, P.M.; Huang, C.; Islam, Z.F.; Schittenhelm, R.B.; King, G.M.; Greening, C. Atmospheric carbon monoxide oxidation is a widespread mechanism supporting microbial survival. ISME J. 2019, 13, 2868-2881. [CrossRef]

16. Patrauchan, M.A.; Miyazawa, D.; LeBlanc, J.C.; Aiga, C.; Florizone, C.; Dosanjh, M.; Davies, J.; Eltis, L.D.; Mohn, W.W. Proteomic analysis of survival of Rhodococcus jostii RHA1 during carbon starvation. Appl. Environ. Microbiol. 2012, 78, 6714-6725. [CrossRef]

17. Berney, M.; Cook, G.M. Unique flexibility in energy metabolism allows mycobacteria to combat starvation and hypoxia. PLoS ONE 2010, 5, e8614. [CrossRef]

18. Islam, Z.F.; Cordero, P.R.F.; Feng, J.; Chen, Y.J.; Bay, S.K.; Jirapanjawat, T.; Gleadow, R.M.; Carere, C.R.; Stott, M.B.; Chiri, E.; et al. Two Chloroflexi classes independently evolved the ability to persist on atmospheric hydrogen and carbon monoxide. ISME J. 2019, 13, 1801-1813. [CrossRef]

19. Hernández, M.; Calabi, M.; Conrad, R.; Dumont, M.G. Analysis of the microbial communities in soils of different ages following volcanic eruptions. Pedosphere 2020, 30, 126-134. [CrossRef]

20. Hernández, M.; Dumont, M.G.; Calabi, M.; Basualto, D.; Conrad, R. Ammonia oxidizers are pioneer microorganisms in the colonization of new acidic volcanic soils from South of Chile. Environ. Microbiol. Rep. 2014, 6, 70-79. [CrossRef]

21. Andrews, S. FastQC: A Quality Control Tool for High throughput Sequence Data 2010. Available online: https://www.bioinformatics.babraham.ac.uk/projects/fastqc/ (accessed on 31 December 2019).

22. Bushnell, B.; Rood, J.; Singer, E. BBMerge-Accurate paired shotgun read merging via overlap. PLoS ONE 2017, 12, e0185056. [CrossRef] [PubMed]

23. Li, D.; Liu, C.M.; Luo, R.; Sadakane, K.; Lam, T.W. MEGAHIT: An ultra-fast single-node solution for large and complex metagenomics assembly via succinct de Bruijn graph. Bioinformatics 2015, 31, 1674-1676. [CrossRef] [PubMed]

24. Bankevich, A.; Nurk, S.; Antipov, D.; Gurevich, A.A.; Dvorkin, M.; Kulikov, A.S.; Lesin, V.M.; Nikolenko, S.I.; Pham, S.; Prjibelski, A.D.; et al. SPAdes: A new genome assembly algorithm and its applications to single-cell sequencing. J. Comput. Biol. 2012, 19, 455-477. [CrossRef] [PubMed]

25. Nurk, S.; Meleshko, D.; Korobeynikov, A.; Pevzner, P.A. metaSPAdes: A new versatile metagenomic assembler. Genome Res. 2017, 27, 824-834. [CrossRef]

26. Mikheenko, A.; Saveliev, V.; Gurevich, A. MetaQUAST: Evaluation of metagenome assemblies. Bioinformatics 2016, 32, 1088-1090. [CrossRef]

27. Ondov, B.D.; Bergman, N.H.; Phillippy, A.M. Interactive metagenomic visualization in a Web browser. BMC Bioinform. 2011, 12, 385. [CrossRef]

28. Uritskiy, G.V.; DiRuggiero, J.; Taylor, J. MetaWRAP-a flexible pipeline for genome-resolved metagenomic data analysis. Microbiome 2018, 6, 158. [CrossRef]

29. Wu, Y.W.; Simmons, B.A.; Singer, S.W. MaxBin 2.0: An automated binning algorithm to recover genomes from multiple metagenomic datasets. Bioinformatics 2016, 32, 605-607. [CrossRef]

30. Kang, D.D.; Froula, J.; Egan, R.; Wang, Z. MetaBAT, an efficient tool for accurately reconstructing single genomes from complex microbial communities. PeerJ 2015, 3, e1165. [CrossRef]

31. Alneberg, J.; Bjarnason, B.S.; de Bruijn, I.; Schirmer, M.; Quick, J.; Ijaz, U.Z.; Lahti, L.; Loman, N.J.; Andersson, A.F.; Quince, C. Binning metagenomic contigs by coverage and composition. Nat. Methods 2014, 11, 1144-1146. [CrossRef]

32. Parks, D.H.; Imelfort, M.; Skennerton, C.T.; Hugenholtz, P.; Tyson, G.W. CheckM: Assessing the quality of microbial genomes recovered from isolates, single cells, and metagenomes. Genome Res. 2015, 25, 1043-1055. [CrossRef] [PubMed]

33. Laczny, C.C.; Sternal, T.; Plugaru, V.; Gawron, P.; Atashpendar, A.; Margossian, H.H.; Coronado, S.; van der Maaten, L.; Vlassis, N.; Wilmes, P. VizBin-An application for reference-independent visualization and human-augmented binning of metagenomic data. Microbiome 2015, 3, 1-7. [CrossRef] [PubMed] 
34. Olm, M.R.; Brown, C.T.; Brooks, B.; Banfield, J.F. dRep: A tool for fast and accurate genomic comparisons that enables improved genome recovery from metagenomes through de-replication. ISME J. 2017, 11, 2864-2868. [CrossRef] [PubMed]

35. Zhang, Z.; Schwartz, S.; Wagner, L.; Miller, W. A greedy algorithm for aligning DNA sequences. J. Comput. Biol. 2000, 7, 203-214. [CrossRef] [PubMed]

36. Mizuno, C.M.; Rodriguez-Valera, F.; Kimes, N.E.; Ghai, R. Expanding the marine virosphere using metagenomics. PLoS Genet. 2013, 9, e1003987. [CrossRef]

37. Warnes, G.R.; Bolker, B.; Bonebakker, L.; Gentleman, R.; Huber, W.; Liaw, W.H.A.; Lumley, T.; Maechler, M.; Magnusson, A.; Moeller, S.; et al. Gplots: Various R Programming Tools for Plotting Data. The Comprehensive R Archive Network. 2009. Available online: https://cran.r-project.org/web/packages/gplots/index.html (accessed on 31 May 2020).

38. Hyatt, D.; Chen, G.L.; LoCascio, P.F.; Land, M.L.; Larimer, F.W.; Hauser, L.J. Prodigal: Prokaryotic gene recognition and translation initiation site identification. BMC Bioinform. 2010, 11, 119. [CrossRef]

39. Bose, T.; Haque, M.M.; Reddy, C.; Mande, S.S. COGNIZER: A framework for functional annotation of metagenomic datasets. PLoS ONE 2015, 10, e0142102. [CrossRef]

40. Kanehisa, M.; Sato, Y. KEGG Mapper for inferring cellular functions from protein sequences. Protein Sci. 2020, 29, 28-35. [CrossRef]

41. King, G.M.; Weber, C.F. Distribution, diversity and ecology of aerobic CO-oxidizing bacteria. Nat. Rev. Microbiol. 2007, 5, 107-118. [CrossRef]

42. Aziz, R.K.; Bartels, D.; Best, A.A.; DeJongh, M.; Disz, T.; Edwards, R.A.; Formsma, K.; Gerdes, S.; Glass, E.M.; Kubal, M.; et al. The RAST Server: Rapid annotations using subsystems technology. BMC Genomics 2008, 9, 75. [CrossRef]

43. Overbeek, R.; Olson, R.; Pusch, G.D.; Olsen, G.J.; Davis, J.J.; Disz, T.; Edwards, R.A.; Gerdes, S.; Parrello, B.; Shukla, M.; et al. The SEED and the Rapid Annotation of microbial genomes using Subsystems Technology (RAST). Nucleic Acids Res. 2014, 42, D206D214. [CrossRef] [PubMed]

44. Rodriguez-R, L.M.; Konstantinidis, K.T. The enveomics collection: A toolbox for specialized analyses of microbial genomes and metagenomes. PeerJ Prepr. 2016, 4, e1900v1.

45. Price, M.N.; Dehal, P.S.; Arkin, A.P. FastTree 2-Approximately maximum-likelihood trees for large alignments. PLoS ONE 2010, 5, e9490. [CrossRef] [PubMed]

46. Darling, A.E.; Jospin, G.; Lowe, E.; Matsen, F.A.; Bik, H.M.; Eisen, J.A. PhyloSift: Phylogenetic analysis of genomes and metagenomes. PeerJ 2014, 2, e243. [CrossRef]

47. Jones, D.T.; Taylor, W.R.; Thornton, J.M. The rapid generation of mutation data matrices from protein sequences. Comp. Appl. Biosci. 1992, 8, 275-282. [CrossRef]

48. Kumar, S.; Stecher, G.; Li, M.; Knyaz, C.; Tamura, K. MEGA X: Molecular Evolutionary Genetics Analysis across computing platforms. Mol. Biol. Evol. 2018, 35, 1547-1549. [CrossRef]

49. Stecher, G.; Tamura, K.; Kumar, S. Molecular Evolutionary Genetics Analysis (MEGA) for macOS. Mol. Biol. Evol. 2020, 37, 1237-1239. [CrossRef]

50. King, C.E.; King, G.M. Description of Thermogemmatispora carboxidivorans sp. Nov., a carbon-monoxide-oxidizing member of the class Ktedonobacteria isolated from a geothermally heated biofilm, and analysis of carbon monoxide oxidation by members of the class Ktedonobacteria. Int. J. Syst. Evol. Microbiol. 2014, 64, 1244-1251. [CrossRef]

51. Chang, Y.J.; Land, M.; Hauser, L.; Chertkov, O.; Del Rio, T.G.; Nolan, M.; Copeland, A.; Tice, H.; Cheng, J.F.; Lucas, S.; et al. Non-contiguous finished genome sequence and contextual data of the filamentous soil bacterium Ktedonobacter racemifer type strain (SOSP1-21). Stand. Genomic. Sci. 2011, 5, 97-111. [CrossRef]

52. Zheng, Y.; Wang, C.M.; Sakai, Y.; Abe, K.; Yokota, A.; Yabe, S. Dictyobacter vulcani sp. nov., belonging to the class Ktedonobacteria, isolated from soil of the Mt Zao volcano. Int. J. Syst. Evol. Microbiol. 2020, 70, 1805-1813. [CrossRef]

53. Qin, Q.L.; Xie, B.B.; Zhang, X.Y.; Chen, X.L.; Zhou, B.C.; Zhou, J.; Oren, A.; Zhang, Y.Z. A proposed genus boundary for the prokaryotes based on genomic insights. J. Bacteriol. 2014, 196, 2210-2215. [CrossRef] [PubMed]

54. King, G.M.; Weber, C.F.; Nanba, K.; Sato, Y.; Ohta, H. Atmospheric CO and hydrogen uptake and CO oxidizer phylogeny for Miyake-jima, Japan volcanic deposits. Microbes Environ. 2008, 23, 299-305. [CrossRef] [PubMed]

55. Martinez-Hernandez, F.; Fornas, O.; Lluesma Gomez, M.; Bolduc, B.; de la Cruz Peña, M.J.; Martínez, J.M.; Anton, J.; Gasol, J.M.; Rosselli, R.; Valera, F.R.; et al. Single-virus genomics reveals hidden cosmopolitan and abundant viruses. Nat. Commun. 2017, 8, 15892. [CrossRef] [PubMed] 
56. Ramos-Barbero, M.D.; Martin-Cuadrado, A.B.; Viver, T.; Santos, F.; Martinez-Garcia, M.; Anton, J. Recovering microbial genomes from metagenomes in hypersaline environments: The good, the bad and the ugly. Syst. Appl. Microbiol. 2019, 42, 30-40. [CrossRef] [PubMed]

57. Weber, C.F.; King, G.M. Quantification of Burkholderia coxL genes in Hawaiian volcanic deposits. Appl. Environ. Microbiol. 2010, 76, 2212-2217. [CrossRef]

58. Dunfield, K.E.; King, G.M. Molecular analysis of carbon monoxide-oxidizing bacteria associated with recent Hawaiian volcanic deposits. Appl. Environ. Microbiol. 2004, 70, 4242-4248. [CrossRef]

59. Cavaletti, L.; Monciardini, P.; Bamonte, R.; Schumann, P.; Rohde, M.; Sosio, M.; Donadio, S. New lineage of filamentous, spore-forming, Gram-positive bacteria from Soil. Appl. Environ. Microbiol. 2006, 72, 4360-4369. [CrossRef]

60. Yabe, S.; Sakai, Y.; Abe, K.; Yokota, A.; Také, A.; Matsumoto, A.; Sugiharto, A.; Susilowati, D.; Hamada, M.; Nara, K.; et al. Dictyobacter aurantiacus gen. nov., sp. nov., a member of the family Ktedonobacteraceae, isolated from soil, and emended description of the genus Thermosporothrix. Int. J. Syst. Evol. Microbiol. 2017, 67, 2615-2621. [CrossRef]

61. Yabe, S.; Aiba, Y.; Sakai, Y.; Hazaka, M.; Yokota, A. Thermosporothrix hazakensis gen. nov., sp. nov., isolated from compost and description of Thermosporotrichaceae fam. nov. within the class Ktedonobacteria. Int. J. Syst. Evol. Microbiol. 2010, 60, 1794-1801. [CrossRef]

62. Yabe, S.; Sakai, Y.; Yokota, A. Thermosporothrix narukonensis sp. nov., belonging to the class Ktedonobacteria, isolated from fallen leaves on geothermal soil, and emended description of the genus Thermosporothrix. Int. J. Syst. Evol. Microbiol. 2016, 66, 2152-2157. [CrossRef]

63. Yan, B.; Guo, X.; Liu, M.; Huang, Y. Ktedonosporobacter rubrisoli gen. nov., sp. nov., a novel representative of the class Ktedonobacteria, isolated from red soil, and proposal of Ktedonosporobacteraceae fam. nov. Int. J. Syst. Evol. Microbiol. 2020, 70, 1015-1025. [CrossRef]

64. Wang, C.M.; Zheng, Y.; Sakai, Y.; Toyoda, A.; Minakuchi, Y.; Abe, K.; Yokota, A.; Yabe, S. Tengunoibacter tsumagoiensis gen. nov., sp. nov., Dictyobacter kobayashii sp. nov., Dictyobacter alpinus sp. nov., and description of Dictyobacteraceae fam. nov. within the order Ktedonobacterales isolated from Tengu-no-mugimeshi, a soil-like granular mass of microorganisms, and emended descriptions of the genera Ktedonobacter and Dictyobacter. Int. J. Syst. Evol. Microbiol. 2019, 69, 1910-1918. [PubMed]

65. Zheng, Y.; Wang, C.M.; Sakai, Y.; Abe, K.; Yokota, A.; Yabe, S. Thermogemmatispora aurantia sp. nov. and Thermogemmatispora argillosa sp. nov., within the class Ktedonobacteria, and emended description of the genus Thermogemmatispora. Int. J. Syst. Evol. Microbiol. 2019, 69, 1744-1750. [CrossRef]

66. Yabe, S.; Aiba, Y.; Sakai, Y.; Hazaka, M.; Yokota, A. Thermogemmatispora onikobensis gen. nov., sp. nov. and Thermogemmatispora foliorum sp. nov., isolated from fallen leaves on geothermal soils, and description of Thermogemmatisporaceae fam. nov. and Thermogemmatisporales ord. nov. within the class Ktedonobacteria. Int. J. Syst. Evol. Microbiol. 2011, 61, 903-910. [PubMed]

67. Greening, C.; Biswas, A.; Carere, C.R.; Jackson, C.J.; Taylor, M.C.; Stott, M.B.; Cook, G.M.; Morales, S.E. Genomic and metagenomic surveys of hydrogenase distribution indicate $\mathrm{H} 2$ is a widely utilised energy source for microbial growth and survival. ISME J. 2016, 10, 761-777. [CrossRef] [PubMed]

68. Sato, Y.; Nishihara, H.; Yoshida, M.; Watanabe, M.; Rondal, J.D.; Concepcion, R.N.; Ohta, H. Cupriavidus pinatubonensis sp. nov. and Cupriavidus laharis sp. nov., novel hydrogen- oxidizing, facultatively chemolithotrophic bacteria isolated from volcanic mudflow deposits from Mt. Pinatubo in the Philippines. Int. J. Syst. Evol. Microbiol. 2006, 56, 973-978. [CrossRef] [PubMed]

Publisher's Note: MDPI stays neutral with regard to jurisdictional claims in published maps and institutional affiliations.

(C) 2020 by the authors. Licensee MDPI, Basel, Switzerland. This article is an open access article distributed under the terms and conditions of the Creative Commons Attribution (CC BY) license (http://creativecommons.org/licenses/by/4.0/). 